Documentation et bibliothèques

DOCUMENTATION BIBLIOTHEQUES

Une grande bibliothèque publique

A Large Public Library

Una biblioteca grande y pública

\title{
Hélène Roussel
}

Volume 51, numéro 1, janvier-mars 2005

Bibliothèque nationale du Québec

URI : https://id.erudit.org/iderudit/1030122ar

DOI : https://doi.org/10.7202/1030122ar

Aller au sommaire du numéro

\section{Éditeur(s)}

Association pour l'avancement des sciences et des techniques de la documentation (ASTED)

\section{ISSN}

0315-2340 (imprimé)

2291-8949 (numérique)

Découvrir la revue

\section{Citer cet article}

Roussel, H. (2005). Une grande bibliothèque publique. Documentation et bibliothèques, 51(1), 49-50. https://doi.org/10.7202/1030122ar
Résumé de l'article

La Grande Bibliothèque, qui ouvre ses portes au public ce printemps, regroupe une vaste collection constituée de la Collection nationale québécoise et d'une Collection universelle de prêt et de référence.
Tous droits réservés (C) Association pour l'avancement des sciences et des techniques de la documentation (ASTED), 2005
Ce document est protégé par la loi sur le droit d'auteur. L'utilisation des services d'Érudit (y compris la reproduction) est assujettie à sa politique d'utilisation que vous pouvez consulter en ligne.

https://apropos.erudit.org/fr/usagers/politique-dutilisation/ 


\section{Une grande bibliothèque publique}

HÉLÈNE ROUSSEL

Directrice générale de la diffusion

\section{RÉSUMÉ | ABStRacts | RESUMEN}

La Grande Bibliothèque, qui ouvre ses portes au public ce printemps, regroupe une vaste collection constituée de la Collection nationale québécoise et d'une Collection universelle de prêt et de référence.

\section{A Large Public Library}

The Grande Bibliothèque will open its doors to the public in the spring of 2005. It brings together a vast collection made up of the national collection of Québec and a circulating and reference collection.

Una biblioteca grande y pública

La Biblioteca Grande, que abre sus puertas al público esta primavera, reúne una vasta colección formada por la colección nacional quebequense y otra universal, de préstamo y consulta.

\section{L}

A FRÉQUENTATION DES BIBLIOTHÈQUES et la demande documentaire n'ont cessé de croître ces dernières années et même les institutions les mieux dotées en termes d'espaces et de ressources ont fini par se trouver à l'étroit. Au Québec, en 1997, le rapport Richard a ainsi conclu à la nécessité de bâtir une nouvelle grande bibliothèque publique à Montréal, la Grande bibliothèque du Québec (GBQ), dont la première pierre a été posée le 3 décembre 2001.

La poursuite de la réflexion autour de la rationalisation des missions et de l'optimisation de l'utilisation des ressources a ensuite mené à la fusion entre la GBQ et la BNQ, effective en mars 2002, et à celle annoncée pour l'automne 2005, entre la BNQ et les Archives nationales du Québec. L'ensemble de ce processus de réévaluation a été dominé par l'idée de réseau. Dans le cadre du projet de Grande Bibliothèque, à l'édifice de diffusion de la BNQ, les collections sont constituées dans une perspective de bibliothèque-ressource, accessible à tous et complémentaire aux bibliothèques québécoises. Les services ont été élaborés de telle sorte qu'ils puissent, autant que possible, être dispensés dans l'ensemble du territoire québécois, en prolongement des services rendus par les bibliothèques locales. De plus, en vertu d'une entente spécifique avec la Ville de Montréal, la BNQ prend la relève de la Bibliothèque centrale et de sa Phonothèque auprès des Montréalais et des bibliothèques en arrondissement. La Grande Bibliothèque répond ainsi aux besoins de nombreuses et diverses clientèles : adultes ou jeunes, amateurs ou amateurs avertis, étudiants ou autodidactes, chercheurs, gens d'affaires, nouveaux arrivants, personnes ayant un handicap visuel ou un autre handicap.

\section{UN IMPORTANT REGROUPEMENT DE COLLECTIONS}

La diffusion de documents auprès d'un large public est une activité nouvelle pour la Bibliothèque nationale du Québec. Dans la Grande Bibliothèque, les usagers pourront désormais consulter sur place et librement une collection patrimoniale québécoise de diffusion regroupée dans un seul bâtiment. Ils auront également accès à une vaste collection universelle de prêt et de référence : ils y trouveront des documents sur tous les supports, portant sur tous les sujets et répondant aux besoins de clientèles variées. La 
majorité de ces ressources seront également disponibles à distance, soit par la numérisation, la livraison électronique de documents, ou encore, grâce au réseau de prêt établi entre les bibliothèques québécoises, qu'elles soient publiques, collégiales, universitaires ou autres.

Vaste et unique, la collection de la Grande Bibliothèque comprend quatre millions de documents et est constituée de deux composantes principales, soit :

- la Collection nationale, témoin du patrimoine documentaire édité au Québec ou créé par des Québécois, ou encore, dont le sujet est relatif au Québec;

- la Collection universelle, destinée au prêt et à la référence, répartie sur six étages et regroupée par thématiques.

Dans la Collection nationale, la Grande Bibliothèque offre au public, généralement en accès libre, les collections autrefois disponibles dans les deux édifices de la BNQ qui ont fermé leurs portes au début de l'année 2005 : la bibliothèque Saint-Sulpice et l'édifice Ægidius-Fauteux, ainsi que certaines collections de l'édifice de conservation.

Les journaux, revues et publications gouvernementales de l'édifice Ægidius-Fauteux sont proposés aux usagers dans leur forme originale, mais aussi sur microforme ou en ligne, dans la collection numérique. Les documents transférés de la Bibliothèque centrale de Montréal ont complété la collection des publications gouvernementales québécoises et augmenté significativement celle des publications gouvernementales canadiennes, la BCM ayant été la seule bibliothèque publique québécoise à être dépositaire universel de ces publications, en français et en anglais, depuis 1927.

La collection de prêt et de référence peut compter sur de riches collections de départ et plus particulièrement sur le fonds constitué depuis près de 100 ans par la Bibliothèque centrale de Montréal et, plus récemment, par sa Phonothèque : leurs documents ont été acquis auprès de la Ville de Montréal et transférés à la Grande Bibliothèque. Sont également à la disposition des usagers de la BNQ, les livres en braille et les livres sonores adaptés provenant de l'Institut Nazareth et Louis-Braille et de La Magnétothèque, destinés aux personnes ayant un handicap visuel.

De plus, dès l'ouverture de la Grande Bibliothèque au printemps 2005 , la collection de prêt et de référence aura été enrichie de 475 ooo nouveaux documents ( 350 ooo livres, 125 ooo documents audiovisuels et électroniques et de plusieurs centaines de nouveaux livres adaptés), grâce à un vaste programme d'acquisition destiné à compléter les collections initiales, historiquement victimes de budgets insuffisants pour alimenter une bibliothèque-ressource et de recherche qui doit également répondre à la demande pour les titres populaires et récents.
Dans certains cas, la Collection nationale et la collection de prêt et de référence se rejoignent dans un même ensemble à l'intérieur de la Grande Bibliothèque. Il en est ainsi du Centre québécois de ressources en littérature Jeunesse, logé dans l'Espace Jeunes au niveau du métro, qui regroupera environ 40 ooo documents : 14 ooo livres de la Collection nationale remontant aux débuts de la littérature enfantine y côtoient quelque 26 ooo livres transférés de la BCM, témoins des lectures des jeunes dans les bibliothèques montréalaises de quartier au fil des décennies.

De même, dans un espace dédié à la collection patrimoniale de musique, section musique et films, plus de 13 ooo enregistrements sonores et 4 , ooo imprimés musicaux du Centre de conservation de la BNQ ont rejoint la collection Musique et films de la Grande Bibliothèque, au niveau 4. Et près de 2000 documents électroniques de la collection patrimoniale sont venus enrichir la logithèque.

En matière de généalogie, généalogistes et chercheurs sur les familles québécoises profitent désormais d'un service amélioré par la réunion des collections et des services dans un seul lieu, soit au Centre des archives de Montréal. Ils y retrouvent les documents provenant de la salle Gagnon de la BCM, référence unique et célèbre dans le domaine de la généalogie, voisinant les incontournables documents d'archives pour quiconque effectue une recherche poussée. Une entente entre la Bibliothèque nationale et les Archives nationales du Québec, précédant leur fusion décidée et prévue pour l'automne 2005, a permis de concrétiser la synergie existant entre les deux institutions. Seules quelques rues séparent le Centre des archives de la Grande Bibliothèque, ce qui facilite la tâche aux chercheurs qui ont besoin de compléter leurs recherches en utilisant les ressources de la BNQ. Un lien de télécommunication par fibre optique relie déjà les deux bâtiments.

Quant au Service québécois du livre adapté, né en 2001 du regroupement des services de bibliothèque de La Magnétothèque et de l'Institut Nazareth et LouisBraille, il s'appuie sur les collections transférées de ces deux organismes vers la BNQ. Ce sont environ 50 ooo documents dont 11 ooo titres en braille, 11 ooo sur cassettes et 300 sur disques compacts qui seront proposés aux personnes atteintes de déficiences visuelles. Au cours de l'année 2005, la BNQ ajoutera environ 1 ooo nouveaux titres, en majorité disponibles dès l'ouverture de la Grande Bibliothèque.

On le constate, le regroupement inédit des collections de la BNQ, de la BCM, de l'INLB et de La Magnétothèque et la naissance prochaine de Bibliothèque et Archives nationales du Québec, dotent le Québec d'une institution culturelle riche et unique en Amérique du Nord. (-) 